\section{Atividade física e gestação: saúde da gestante não atleta e crescimento fetal}

\section{Physical activity and pregnancy: non- athletic pregnant women's health and fetal growth}

\author{
Daniele Costa Batista 1 \\ Vera Lucia Chiara 2 \\ Sílvia Angela Gugelmin 3 \\ Patrícia Dias Martins 4
}

\begin{abstract}
1 Pós-graduação de Nutrição e Atividade Física. Instituto de Nutrição. Universidade do Estado do Rio de Janeiro. 2-4 Departamento de Nutrição Social. Instituto de Nutrição. Universidade do Estado do Rio de Janeiro. Rua São Francisco Xavier, 524, $12^{\circ}$ andar, sala 12.001, Bloco D. Rio de Janeiro, RJ, Brasil. CEP: 20.559-900.
\end{abstract}

\begin{abstract}
The benefits of physical activity on the life cycle are recognized but there is no consensus of physical activity when related to pregnancy. This article reviews aspects and effects of the practice of physical activities during pregnancy on non-athletic pregnant women and on the fetus. Medline and Pubmed were referred to in the period of 1992-2002. Among the benefits, the articles highlights: prevention and reduction of backaches, pains on hands and feet and cardiovascular stress, strengthening of pelvic muscles, reduction of premature deliveries and $C$-sections, more flexibility and tolerance to pain, ponderal gain control and increase of the self-esteem in pregnant women. As for the fetus there was improvement of weight and nutritional conditions. There were no recommendations of nutritional needs for non-athletic pregnant women. There was no consensus related to nutritional needs and the type of physical activities for pregnant women. Physical exercises in the water were considered more adequate. There are various counter-indications for exercises during pregnancy. The conclusion is that regular and moderate physical and controlled activities in the beginning of pregnancy are beneficial to mothers and fetuses.
\end{abstract}

Key words Motor activity, Exercise, Pregnancy; Embryo and fetal development
Resumo São reconhecidos os benefícios da prática de atividade física sobre o ciclo vital, sem consenso quanto à gestação. Este artigo reviu considerações e efeitos da prática da atividade física durante a gestação sobre a saúde da gestante não-atleta e o feto. Foram utilizados os bancos de dados Medline e Pubmed referentes ao periodo 1992-2002. Dentre os beneficios, os artigos destacam: prevenção e redução de lombalgias, de dores das mãos e pés e estresse cardiovascular, fortalecimento da musculatura pélvica, redução de partos prematuros e cesáreas, maior flexibilidade e tolerância à dor, controle do ganho ponderal e elevação da auto-estima da gestante. No feto, observou-se aumento do peso ao nascer e melhoria da condição nutricional. Não se encontraram recomendações de necessidades nutricionais para a gestante não-atleta. Não existiu consenso quanto às necessidades nutricionais e o tipo de atividade física dessa gestante, sendo o exercicio na água o mais indicado. Porém destacaram-se diversas contra-indicações da prática de exercícios na gestação. Concluiu-se que a atividade física, sendo regular, moderada e controlada desde o inicio da gestação, promove benefícios para a saúde materna e fetal.

Palavras-chave Atividade motora, Exercicio, Gravidez, Desenvolvimento embrionário e fetal 


\section{Introdução}

A atividade física é definida como qualquer movimento corporal decorrente de contração muscular, com dispêndio energético acima do repouso que, em última análise, permite o aumento da força física, flexibilidade do corpo e maior resistência, com mudanças, seja no campo da composição corporal ou de performance desportiva. ${ }^{1}$ A prática de atividade física regular demonstra a opção por um estilo de vida mais ativo, relacionado ao comportamento humano voluntário, onde se integram componentes e determinantes de ordem biológica e psico-sócio-cultural.

Evidências baseadas em estudos epidemiológi$\cos 2,3$ confirmaram o papel decisivo da prática da atividade física na promoção da saúde, na qualidade de vida e na prevenção e/ou controle de diversas doenças. Diretrizes para a promoção de estilos de vida saudáveis têm sido recomendadas por órgãos envolvidos com a saúde pública, destacando-se a prática de atividade física regular em todo o ciclo vital. 4 A World Health Organization (WHO $)^{5}$ estabelece que para prevenir e manejar a atual epidemia global de obesidade, deve haver a integração da atividade física regular no cotidiano, acompanhada de melhoria da qualidade de vida e alimentação.

Privilégio anteriormente do sexo masculino, as mulheres passaram, recentemente, a representar importante grupo na prática da atividade física e, embora persistam controvérsias sobre esta prática no período gestacional, a atividade física vem se integrando de forma crescente nesse grupo. Em décadas passadas, as gestantes eram aconselhadas a reduzirem suas atividades e interromperem, até mesmo, o trabalho ocupacional, especialmente durante os estágios finais da gestação, acreditando-se que o exercício aumentaria o risco de trabalho de parto prematuro por meio de estimulação da atividade uterina. 6

No entanto, em meados da década de 90, o American College of Obstetricians and Gynecologists (ACOG), ${ }^{7}$ reconheceu que a prática da atividade física regular no período gestacional, deveria ser desenvolvida desde que a gestante apresentasse condições apropriadas. Segundo Artal e Gardin, 6 as mudanças na sociedade quanto aos direitos de cidadania relativos à posição das mulheres, que se fortaleceram através de movimentos sociais nos anos sessenta, refletiram-se também na assistência pré-natal, ampliando as estratégias implementadas no atendimento, o que contribuiu para que o exercício físico ganhasse ênfase. Todavia, para Gallup, 8 ainda são insuficientes até hoje, os trabalhos publicados com enfoque na prática da atividade física regular durante a gestação bem como informações sobre a sua indicação.
O presente trabalho tem por objetivo apresentar, através de revisão de literatura, considerações levantadas a respeito da prática de atividade física regular durante a gestação, com enfoque especial sobre o efeito na saúde da gestante não atleta e no crescimento fetal. A revisão dos artigos limitou-se ao período de 1992 a 2002, utilizando-se os termos: exercise, exercise and pregnancy, physical activity, pregnancy and physical activity. As publicações foram obtidas nos bancos de dados Medline e Pubmed, sendo consultados ainda livros didáticos de diferentes períodos, para esclarecimentos sobre definições de atividade física, alterações orgânicas durante a gestação e crescimento fetal.

\section{Aspectos nutricionais}

Durante a gestação o estado anabólico permanece dinâmico em função das demandas nutricionais, promovendo ajustes contínuos em relação a diversos nutrientes e micronutrientes. $\mathrm{O}$ ganho de peso que no início do período gestacional é reduzido comparado à fase final, necessita ser permanentemente controlado para evitar a ocorrência de deficiência ou excesso. O ganho de peso em excesso pode expor a gestante ao desenvolvimento de diversas patologias, tais como hipertensão arterial, diabetes, obesidade pósparto, macrossomia fetal, além de complicações no parto e puerpério. ${ }^{9}$ A deficiência do ganho de peso pode trazer prejuízo para o crescimento e desenvolvimento fetal. No entanto, o ganho de peso insuficiente extrapola este único aspecto, sendo prejudicial para a tríade gestante, trabalho de parto e feto, especialmente frente à prática de atividade física regular. 10

Nos tempos atuais, o controle do ganho de peso gestacional, adquiriu um "novo conceito" com o impingir social da "estética da magreza". A preocupação da gestante, quanto ao ganho de peso, dividese entre o peso necessário e suficiente que deverá obter sem prejuízo para a gestação, e o peso que terá após o parto. 9 Todavia, vale lembrar, a dieta de controle para perda ou manutenção de peso não é indicada nessa fase, mesmo para gestantes obesas, praticantes ou não de atividade física. 9,10

Segundo estudo de Dewey e McCrory, ${ }^{11}$ ainda não existe consenso sobre a relação entre o ganho de peso gestacional e a prática de exercícios físicos. Para Clapp e Little, 3 tal associação foi observada em um estudo longitudinal com 79 mulheres praticantes de atividade física recreacional, sendo subdivididas em 44 que permaneceram praticando exercícios físicos anterior e durante a gestação e 35 que reduziram 
(4) ou cessaram (31) a atividade quando gestantes. Os autores destacaram que aquelas que praticaram a atividade física durante o terceiro trimestre de gestação apresentaram redução na velocidade de ganho de peso com significante diminuição da gordura localizada, aferida através de cinco dobras subcutâneas ( $22 \pm 2 \mathrm{~mm}$ em praticantes e $31 \pm 2 \mathrm{~mm}$ em não praticantes). $\mathrm{O}$ estudo também revelou que as mulheres com melhor nível de educação e não fumantes foram mais motivadas a manterem dieta balanceada, iniciando a gestação com o peso adequado e acumulando menos gordura no último trimestre da gravidez.

Resultado diferenciado foi observado no estudo descritivo de Horns et al.,12 comparando primíparas sedentárias (48) e praticantes de atividade física regular (53) durante o último trimestre de gravidez. Os autores concluíram que a prática da atividade física não apresentou significante efeito sobre o ganho de peso materno, mas associou-se $\left(\chi^{2}=7,45\right.$ e $\left.p<0,01\right)$ com a redução de edema, cãibra nas pernas e fadiga. Uma questão metodológica em ambos os estudos refere-se à ausência de relato sobre controle de variáveis que interferem no ganho de peso gestacional, como estado nutricional da gestante, idade, paridade e intervalo interpartal, dentre outros.

Todavia, a American College of Obstetricians and Gynecologists (ACOG) ${ }^{7}$ reconhece que a prática regular de atividade física durante a gestação parece atuar no controle do ganho de peso nesse período e no pós-parto, especialmente quando o suprimento nutricional está adequado. Na mesma linha de interesse sobre o suprimento nutricional na gestação e a prática de atividade física, King, 13 revendo estudos sobre a fisiologia durante a gestação, destacou que as necessidades calóricas durante a prática de atividade física são contra-balanceadas através da compensação entre o tempo despendido na atividade, o tempo de descanso e o gasto energético consumido no tipo de atividade. Para a autora, o gasto energético está diretamente relacionado com a proporção em que varia o aumento da atividade e o aumento do metabolismo basal. Segundo Butterfield e King, 14 esse fenômeno pode apontar para a possibilidade de uma adaptação fisiológica preservando a energia que já esta sendo estimulada. No entanto, os autores têm cautela quanto a essa afirmação e reconhecem a necessidade de que sejam estabelecidas recomendações nutricionais efetivas para gestantes praticantes de atividade física. O suprimento nutricional balanceado e adequado representa o eixo central para a prática de atividade física regular, durante ou fora do período gestacional.

Embora muito se saiba sobre a nutrição do atleta $^{10}$ e a nutrição durante a gravidez, 15 ainda são escassos os estudos integrando os dois aspectos. 14 Considerada-se fundamental que diversos fatores relacionados às necessidades nutricionais na fase gestacional sejam observados, tais como: idade, paridade, intervalo interpartal, estado nutricional préconcepção e atual, atividade ocupacional, tipo e intensidade do exercício físico, e fase gestacional dentre outros. 10

Para as mulheres ativas fisicamente mas nãogestantes, as recomendações de proteína atingem $12 \%$ das calorias totais, enquanto para os lipídios ainda não há consenso, sendo comum empregar-se no máximo $30 \%$ do valor energético total. Dietas com baixo teor de lipídios são inadequadas ao bom rendimento físico. Os carboidratos, utilizados em forma de glicogênio e glicose, são elementos fundamentais na prática da atividade física. Sugere-se que a alimentação de uma mulher ativa fisicamente mas não-gestante, apresente cerca de $60 \%$ das calorias totais em forma de carboidratos, 16 garantindo-se ainda, o suprimento de vitaminas e minerais. A atividade física eleva por diversas vias a necessidade de alguns micronutrientes. O estresse metabólico, por exemplo, exige a adequação especialmente de vitaminas do complexo B,17 sendo indispensável a suplementação de ácido fólico. 18 Recomendações nutricionais são de extrema utilidade como base para orientações e avaliações, embora ainda sejam escassos os estudos com gestantes de países em desenvolvimento. 12 O American College of Sports Medicine, em conjunto ao American Dietetic Association e Dietitians of Canada, 10 consideram que as necessidades nutricionais serão sempre individuais, estando diretamente relacionadas às condições de saúde/nutrição e ao tipo de atividade desenvolvida durante a gestação.

\section{Condições físicas e funcionais da gestante durante a atividade física}

Os benefícios da prática de atividades físicas durante a gestação são diversos e atingem diferentes áreas do organismo materno. O exercício reduz e previne as lombalgias, devido à orientação da postura correta da gestante frente à hiperlordose que comumente surge durante a gestação, em função da expansão do útero na cavidade abdominal e o conseqüente desvio do centro gravitacional. Nestes casos, o exercício físico contribuirá para adaptação de nova postura física, refletindo-se em maior habilidade para a gestante durante a prática da atividade física e do trabalho diário. 19

Na ocorrência de dores nas mãos e membros in- 
feriores, que geralmente acontece por volta do terceiro trimestre, frente a diminuição da flexibilidade nas juntas, a prática da atividade física regular direcionada durante a gestação terá o efeito de minimizálas, possivelmente, por promover menor retenção de líquidos no tecido conectivo. ${ }^{19}$

Sabe-se que a atividade cardiovascular durante a gestação se eleva comparada ao período não gestacional. No entanto, com a prática regular de exercícios físicos reduz-se esse estresse cardiovascular, o que se reflete, especialmente, em freqüências cardíacas mais baixas, maior volume sangüíneo em circulação, maior capacidade de oxigenação, menor pressão arterial, prevenção de trombose e varizes, e redução do risco de diabetes gestacional. 2

Em gestantes que apresentam diabetes gestacional, a atividade física pode contribuir para manter os níveis glicêmicos normais. Essa patologia ocorre, de modo geral, na fase tardia da gestação. 2 Todavia, parece que o excesso de peso corporal pode ser um elemento contrário aos benefícios da atividade física para a gestante. Segundo Dye et al., ${ }^{20}$ que estudaram a prática da atividade física durante a gestação como forma de prevenção de diabetes gestacional, somente depois de controlado o efeito do Índice de Massa Corporal (IMC), foi encontrada associação entre a prática de atividade física e o não surgimento de diabetes gestacional (odds ratio $=1,9$ ). Os autores concluíram que o peso expresso através do IMC foi determinante para o surgimento da patologia, especialmente quando o IMC superava $33 \mathrm{~kg} / \mathrm{m}^{2}$. Em gestantes que apresentaram diabetes gestacional e praticaram atividade física regular durante todo o período gestacional observou-se antecipação no trabalho de parto, quando comparadas com aquelas que interromperam a atividade ao final do segundo semestre. 20

A prática de atividade também trouxe benefícios para gestantes já portadoras de diabetes mellitus sob controle. Jovanik-Peterson e Peterson, 21 ao estabelecerem um programa baseado na dieta e atividade física para gestantes diabéticas, afirmaram que embora ainda seja controversa essa questão, evidências acumuladas na literatura têm demonstrado que sob controle dietético e de atividade física, os níveis glicêmicos podem ser mantidos normais, mesmo em gestantes já diabéticas. Os exercícios físicos praticados durante a gestação devem ser cuidadosamente observados para esse grupo de gestantes, especialmente na fase final, uma vez que têm efeito positivo no amadurecimento cervical e na atividade de contração uterina. 5,21

As vantagens da atividade física durante a gestação se estendem ainda, aos aspectos emocionais, contribuindo para que a gestante torne-se mais autoconfiante e satisfeita com a aparência, eleve a autoestima e apresente maior satisfação na prática dos exercícios. 19

\section{Trabalho de parto e prematuridade}

A literatura consultada abordou três aspectos principais na relação entre atividade física e trabalho de parto: risco de parto prematuro, facilidade do trabalho de parto com melhor recuperação pós-parto e redução do número de cesáreas.

Acreditava-se que a prática da atividade física durante a gestação pudesse estimular indiscriminadamente a contração uterina, promovendo a antecipação do trabalho de parto. 5 Todavia, parece haver consenso de que a prática de atividade física monitorada durante a gestação não contribui para a prematuridade.22-23 Sternfeld, 22 consultando diversos estudos, argumentou que o efeito da estimulação da noradrenalina, que ocorre com a atividade física, pode ser neutralizado tanto com o aumento de catecolaminas nas gestantes, como através dos níveis de catecolaminas fetais que permanecem estáveis à estimulação da noradrenalina materna, protegendo o feto do excesso de atividade uterina.

Bishop et al.,24 também são de opinião contrária ao efeito de prematuridade, acreditando que a atividade física regular fortalece a musculatura pélvica, sendo mais um fator a proporcionar nascimentos a termo. Para esse autores, a idade, paridade e aderência ao programa assistencial são fatores que desempenham papel decisivo no risco para partos prematuros. Para Dye e Oldenettel, 25 o monitoramento constante das condições físicas das gestantes durante o desenvolvimento das atividades físicas regulares, pode cumprir importante papel na determinação da idade gestacional. Os autores basearam esta afirmativa em estudo epidemiológico de revisão de literatura desde 1990 .

Bungum et al. 26 estudando mulheres nulíparas, observaram que gestantes sedentárias apresentaram risco 4,5 vezes maior de nascimentos por cesárea do que as gestantes ativas fisicamente, quando controlada as variáveis idade, tipo de anestesia, alterações no IMC anterior à gravidez, trabalho de parto induzido, e o tipo de hospital de nascimento. Os resultados demonstraram que a participação em exercícios físicos, especialmente nos dois primeiros trimestres, esteve associada efetivamente ao menor risco de cesáreas.

Outros aspectos relacionados aos benefícios da atividade física sobre o trabalho de parto, referem-se 
às demais alterações endócrinas ocorridas durante a gravidez, que se refletem nas articulações e ligamentos pélvicos, promovendo maior flexibilidade. $\mathrm{O}$ aumento de estrogênio contribui para o relaxamento muscular facilitando o parto, suavizando as cartilagens e elevando o fluído sinovial com resultados no alargamento das juntas, facilitando a passagem do feto. ${ }^{8} \mathrm{~A}$ atividade física durante a gestação diminui as dores do parto, contribuindo para que as gestantes fisicamente ativas tolerem melhor o trabalho de parto, principalmente os mais prolongados, do que aquelas não treinadas ou do que aquelas que se exercitavam apenas esporadicamente. 19

A associação do exercício físico durante a gestação e o aborto espontâneo, foi explicada no estudo de Latka et al. 27 como conseqüência do tipo de exercício praticado, que apresentava características de intensidade moderada. À mesma conclusão chegou El-Metwalli et al.,28 que através de caso-controle com 562 gestantes (casos) que tiveram aborto espontâneo e 1.762 gestantes (controles) com gestação à termo. Para os autores, não é a prática de atividade física regular que se associa à prematuridade, e sim a intensidade e o excesso de atividade, tanto em forma de exercícios físicos quanto de atividade ocupacional.

O estudo desenvolvido por Misra et al.,29 com uma coorte randomizada de mulheres de baixa renda praticantes de atividade física, e grupo de comparação constituído por gestantes atuantes apenas em atividades ocupacionais domésticas, revelou que as gestantes não praticantes de atividade física apresentaram maior risco de redução do período gestacional (odds ratio $=1,60$ ), enquanto a prática de exercícios físicos presente no outro grupo de gestantes foi considerada fator de proteção. A World Health Organization (WHO) 30 já alertou que grávidas trabalhando com levantamento freqüente de cargas pesadas, ou seja, em atividade ocupacional intensa, incrementam de 20 a $30 \%$ o risco de parto prematuro.

\section{Atividade física e crescimento fetal}

Os termos crescimento e desenvolvimento referemse às mudanças que ocorrem no complexo fisiológico durante a concepção, a embriogênese e toda a vida fetal. O ambiente materno é decisivo para o crescimento e o desenvolvimento fetal, podendo influencia-los positiva ou negativamente. Dentre os principais fatores de risco que afetam o crescimento fetal destacam-se: primíparas com idade superior a 35 anos, gestante adolescente, baixa escolaridade e renda, história de problemas obstétricos, deficiência nutricional, patologias diversas e dependência química.16 Quanto aos efeitos de risco ou proteção da prática de atividade física durante a gestação sobre o crescimento fetal, existem controvérsias, encontrando-se relato de peso normal, baixo peso e aumento de peso.

Kardel e Kase ${ }^{31}$ analisando o desenvolvimento e o crescimento fetal de bebês nascidos de 42 gestantes saudáveis, subdivididas em dois grupos (intensidade alta e intensidade moderada), não encontraram qualquer diferença entre os grupos quanto ao peso de nascimento e a escala de Apgar. Os autores concluíram, que o estado de saúde e o bom condicionamento das gestantes podem ter desempenhado papel fundamental para este resultado.

No estudo de Hatch et al.,32 não foram encontrados bebês macrossômicos, porém observou-se que as gestantes que se exercitaram durante todos os três trimestres da gestação, tenderam a ter bebês com peso maior do que aquelas que eram sedentárias ou as que iniciaram atividade física já no segundo ou no terceiro trimestre. Esse estudo de coorte incluiu 800 gestantes sob assistência pré-natal. Para a análise, os autores, subdividiram o grupo em três subgrupos: praticantes com intensidade leve/moderada, praticantes com intensidade alta e não praticantes de atividade física. Ajustando os dados por idade gestacional e peso ao nascer, o condicionamento físico anterior à gestação foi altamente relevante para o bom crescimento fetal, tendo em vista que o grupo de gestantes que praticaram exercícios de intensidade alta, apresentou aumento de peso fetal em torno de 300 gramas, enquanto aquelas que não praticaram atividade física, o aumento de peso foi próximo de 100 gramas.

Clapp et al.,33 em estudo experimental randomizado com gestantes não praticantes de atividade física, subdivididas em um grupo experimental (24 mulheres) com prática de exercícios de intensidade moderada três a cinco vezes por semana, e um grupo de comparação (22 mulheres) desenvolvendo algum tipo de exercício recreacional, concluiram que, efetivamente, o exercício físico orientado durante toda a gestação, ou parte da mesma, pode contribuir tanto para o aumento do peso do feto, quanto para sua percentagem de gordura e circunferência craniana. Os autores observaram que para esses resultados destacaram-se outros fatores: a programação dos exercícios sob controle; a adequação dos exercícios às características morfométricas (medidas corporais) das gestantes e o aumento do consumo de diversos nutrientes no último trimestre gestacional.

Em estudo com 2.828 mulheres residentes em Missouri (Estados Unidos da América) que tiveram 
filhos de dezembro de 1989 a março de 1991, Schramm et al.,34 analisaram a relação entre prática de exercícios físicos e peso de nascimento. Os resultados apontaram que a prática de exercícios físicos pode ter desempenhado função protetora (odds ratio $=0,88$ ), uma vez que estas mulheres tiveram filhos com peso igual ou superior a 2.500gramas, dentre os 794 nascidos vivos, contra aquelas que não praticaram exercícios e tiveram filhos com peso inferior a 1.500 gramas, dentre os 782 nascidos vivos. $\mathrm{O}$ estudo excluiu 450 mulheres que tiveram fetos nascidos mortos. Esse resultado merece ser avaliado cuidadosamente tendo em vista que se trata de grande amostra e o intervalo de confiança do odds ratio incluiu a unidade.

Gottlieb $^{35}$ destacou que as contradições nos resultados das pesquisas podem ser inerentes às metodologias empregadas, especialmente no que se refere ao tipo de estudo e ao grupo amostral. O autor apontou que, por vezes, as pesquisas não apresentaram grupo de comparação ou os grupos revelavam importantes distinções entre si, capazes de interferirem nos resultados. Segundo Dye e Oldenettel, 25 análises baseadas em grupos especiais, como atletas de elite, podem ocasionar erro na sua comparação com grupos de gestantes sedentárias. $\mathrm{O}$ cuidado com a metodologia e a análise estatística dos dados em estudos controlados, permitiria maior segurança nas conclusões da relação entre a prática da atividade física e a gestação.

\section{Atividades físicas recomendadas durante a gestação}

Ainda não existem recomendações padronizadas de atividade física durante a gestação. No entanto, frente à ausência de complicações obstétricas, o American College of Obstetricians and Gynecologists, 36 recomendou que a atividade física desenvolvida durante a gestação, tenha por características exercícios de intensidade regular e moderada, com o programa voltado para o período gestacional em que se encontra a mulher, com as atividades centradas nas condições de saúde da gestante, na experiência em praticar exercícios físicos e na demonstração de interesse e necessidade da mesma.

Alguns tipos de atividades físicas como exercícios leves na água, caminhada e bicicleta, já vêm se destacando como prática de atividade física durante o período gestacional. Para Katz,37 a natação é a mais recomendada para a gestante, devido à propriedade inerente do corpo na água, isto é, a flutuabilidade. A atividade física na água é benéfica para os joelhos e geralmente é mais relaxante que outros tipos de exercícios, especialmente os exercícios de força como a musculação. A natação, reduz ainda a freqüência de edema que é um efeito comum na gestação, porém desconfortável. O efeito da água fria sobre o corpo serve também como termorregulador, proporcionando ao feto a possibilidade de maior estabilidade frente à elevação de temperatura e a subsequente diminuição do suprimento de sangue. A temperatura ideal da água deve ficar entre $28^{\circ} \mathrm{C}$ e $30^{\circ} \mathrm{C} .37,38$

\section{Atividades físicas não recomendadas durante a gestação}

Alguns exercícios físicos merecem recomendações especiais sobre o desenvolvimento de sua prática ou contra-indicação neste período. A intensidade do exercício deve ser monitorada de acordo com os sintomas que a gestante apresentar. Esta intensidade se revela através da demanda sobre o sistema cardiovascular. ${ }^{36}$ A relação a seguir apresenta alguns tipos de exercícios físicos e/ou situações não recomendadas para a prática durante o período gestacional: a) qualquer atividade competitiva, artes marciais ou levantamento de peso; 6 b) exercícios com movimentos repentinos ou de saltos, que podem levar a lesão articular;6 c) flexão ou extensão profunda deve ser evitada pois os tecidos conjuntivos já apresentam frouxidão; 6 exercícios exaustivos e/ou que necessitam de equilíbrio principalmente no terceiro trimestre; 39 d) basquetebol e qualquer outro tipo de jogo com bolas que possam causar trauma abdominal;39 e) pratica de mergulho (condições hiperbáricas levam a risco de embolia fetal quando ocorre a descompressão;39 f) qualquer tipo de ginástica aeróbica, corrida ou atividades em elevada altitude são contraindicadas ou, excepcionalmente aceitas com limitações, dependendo das condições físicas da gestante; ${ }^{40} \mathrm{~g}$ ) exercícios na posição supino após o terceiro trimestre podem resultar em obstrução do retorno venoso. 25

\section{Contra-indicações de saúde para prática de atividade física durante a gestação}

Alguns sinais ou sintomas representam sinal de perigo de complicações na gestação durante a prática de atividade física e indicam que o exercício deve ser imediatamente interrompido por constituirem grande risco para a saúde tanto da gestante e quanto do feto. Os principais sinais de que a atividade física deve 
cessar são: perda de líquido aminiótico, dor no peito, sangramento vaginal, enxaqueca, dispnéia, edema, dor nas costas, náuseas, dor abdominal, contrações uterinas, fraquezas musculares e tontura, redução dos movimentos do feto. 25

Mulheres fumantes têm contra-indicação na prática de atividade física em altitudes acima de $2.500 \mathrm{~m} .{ }^{38,39}$ A presença de náuseas, sonolência e desconforto, podem sugerir que o tipo, intensidade, duração e/ou freqüência da atividade física para a gestante devam ser modificados, sem que seja necessário interrompe-la. ${ }^{8}$ A própria prática de atividade física durante a gestação possibilita a ocorrência de fenômenos que devem alertar os especialistas para uma eventual interrupção dos exercícios. Tais sinais correspondem a: dor de qualquer tipo, injúrias musculo-esqueléticas; complicações cardiovasculares; trabalho de parto prematuro; aumento do risco de aborto no primeiro trimestre da gestação e grave hipoglicemia, indicando, igualmente, a interrupção do exercício. 36

A atividade física para gestantes apresenta contraindicação absoluta em mulheres portadoras de: doença cardíaca com alterações hemodinâmicas significativas, doença pulmonar restritiva, multípara com risco de prematuridade, placenta prévia depois de 26 semanas de gestação, ruptura de membranas, sangramento uterino persistente no segundo ou terceiro trimestre, cervix incompetente e pré-eclâmpsia. 34
Considera-se como contra-indicação relativa à prática de exercícios aeróbicos durante a gestação, a presença das seguintes patologias: anemia, arritmia cardíaca materna, diabetes Mellitus tipo I não controlada, bronquite crônica, obesidade mórbida, baixo peso com Índice de Massa Corporal inferior a 12, estilo de vida extremamente sedentário, retardo no crescimento intra-uterino na gestação atual, hipertensão mal controlada, limitações ortopédicas, tabagismo e hipertireoidismo não controlado. ${ }^{36}$

\section{Conclusões}

Embora já se reconheça a contribuição da prática da atividade física regular e orientada durante a gestação, ainda não existe consenso no estabelecimento da conduta ideal para essa prática. Não se encontrou na literatura revista, qualquer tipo de padronização de atividade recomendada por órgãos especializados. Cada autor estabeleceu o tipo de atividade de interesse no estudo, sua duração, intensidade e freqüência, dificultando assim a comparação dos resultados encontrados nos diferentes artigos. Todavia, tendo por base a revisão, concluiu-se que quando indicada, a prática de atividade física regular, moderada, controlada e orientada pode produzir efeitos benéficos sobre a saúde da gestante e do feto.

\section{Referências}

1. Ramos AT. Atividade física: diabéticos, gestantes, terceira idade, crianças e obesos. Rio de Janeiro: Sprint; 1999.

2. Helmrich SP, Ragland DR, Paffenbarger SR. Prevention of non-insulin-dependent diabetes mellitus with physical activity. Med Sci Sports Exerc 1994; 25: 824-30.

3. Clapp JF, Litlle KD. Effect of recreational exercise on pregnancy weight gain and subcutaneous fat deposition. Med Sci Sports Exerc 1995; 27: 170-7.

4. Ministério da Saúde. Áreas técnicas. Projetos de promoção a saúde. Disponível em: www.saude.gov.br. [2001 9 jul]

5. WHO (World Health Organization). Maternal anthropometry and pregnancy outcomes: a WHO collaborative study. Bull World Health Org. 1995; 73: 1-98.

6. Artal R, Gardin SK. Perspectiva histórica. In: Artal R, Wiswell AR, Drinkwater LR. O exercício na gravidez. São Paulo: Manole; 1999. p.1-7.

7. ACOG (American College of Obstetricians and Gynecologists). Exercise during pregnancy and the postpartum period. Washington (DC): O College: 1994.

8. Gallup E. Aspectos legais da prescrição de exercícios para a gravidez. Seção III: Aplicações práticas. In: Artal R, Wiswell AR, Drinkwater LR. O exercício na gravidez.
São Paulo: Manole; 1999. p. 293-8.

9. Rössner S. Physical activity and prevention and treatment of weight gain associated with pregnancy: current evidence and research issues. Med Sci Sports Exerc 1999; 4 Suppl: 560- 63.

10. American College of Sports Medicine, American Dietetic Association, Dietitians of Canada. Nutrition and athletic performance. Med Sci Sports Exerc 2000; 32: 2130-45.

11. Dewey KG, McCrory MA. Effects of dieting and physical activity on pregnancy and lactation. Am J Clin Nutr 1994; 59 Suppl 2: 446-53.

12. Horns PN, Ratcliffe LP, Leggett JC. Pregnancy outcomes among active and sedentary primiparous women. J Obstet Gynecol Neonatal Nurs1996; 25: 49-54.

13. King J. Physiology of pregnancy and nutrient metabolism. Am J Clin Nutr 2000; 71 Suppl 5: 1218-25.

14. Butterfield G, King J. Necessidades nutricionais de gestantes fisicamente ativas. Seção I: Adaptações fisiológicas à gravidez. In: Artal R, Wiswell AR, Drinkwater LR. O exercício na gravidez. São Paulo: Manole; 1999. p. 109-22.

15. Williams SR. Fundamentos de nutrição e dietoterapia. Por- 
to Alegre: Artes Médicas; 1997.

16. Butte NF. Carbohydrate and lipid metabolism in pregnancy: normal compared with gestational diabetes mellitus. Am J Clin Nutr 2000; 71 Suppl 5:1256-61.

17. Manore MM. Effect of physical activity on thiamine, riboflavin, and vitamin B6 requirements. Am J Clin Nutr 2000; 72 Suppl 2: 596-8.

18. Bailey LB. New standard for dietary folate intake in pregnant women. Am J Clin Nutr 2000; 71 Suppl 5: 1304-7.

19. Hartmann S, Bung P. Physical exercise during pregnancy: physiological considerations and recommendations. J Perinat Med 1999; 27: 204-15.

20. Dye TD, Knox LK, Artal R, Aubry RH, Wojtowycz MA Physical activity, obesity, and diabetes in pregnancy. Am J Epidemiol 1997; 146: 961-5.

21. Jovanik-Peterson L, Peterson CM. Exercise and the nutritional mangement of diabetes during pregnancy. Obstet Gynecol Clin North Am 1996; 23: 75-86.

22. Sternfeld B. Physical activity and pregnancy outcome: review and recommendations. Sports Med 1997; 23: 33-47.

23. Lederman SA. Pregnancy weight gain and postpartum loss: avoiding obesity while optimizing the growth and development of the fetus. J Am Med Womens Assoc 2001; 56: 53-8.

24. Bishop KR, Dougherty M, Mooney R, Gimotty P, Williams B. Effects of age, parity, and adherence on pelvic muscle response to exercise. J Obstet Gynecol Neonatal Nurs 1992; 21: 401-6.

25. Dye TD, Oldenettel D. Physical activity and risk of preterm labor: an epidemiological review and synthesis of recent literature. Semin Perinatol 1996; 20: 334-9.

26. Bungum TJ, Peaslee DL, Jackson AW, Perez MA. Exercise during pregnancy and type of delivery in nulliparae. $\mathbf{J}$ Obstet Gynecol Neonatal Nurs 2000; 29: 258-64.

27. Latka M, Kline J, Hatch M. Exercise and spontaneous abortion of Know Karyiotype. Epidemiol Rev 1999;10: 73-5.

28. El-Metwalli AG, Badawy AM, El-Baghdadi LA, El-Wehady A. Occupational physical activity and pregnancy outcome. Eur J Obstet Gynecol Reprod Biol 2001; 100:
41-5.

29. Misra DP, Strobino DM, Stashinko EE, Nagey DA, Narda S. Effects of physical activity on preterm birth. Am J Epidemiol 1998; 147: 628-5.

30. WHO (World Health Organization). Pregnant and lactation women: report of the expert committe on physical status: the use and interpretation of antropometry. Geneve: The Organization; 1995.

31. Kardel RK, Kase T. Training in pregnant women: effects on fetal development and birth. Am J Obstet Gynecol 1998; 178: 280-6.

32. Hatch MC, Shu XO, Mclean DE Maternal exercise during pregnancy, physical fitness, and fetal growth. Am J Epidemiol 1993; 137: 1105-14.

33. Clapp JF, Kim H, Burciu B, Lopez B. Begining regular exercise in early pregnancy: effect on fetoplacental growth. Am J Obstet Gynecol 2000; 183: 1484-8

34. Schramm WF, Stockbauer JW, Hofman HJ. Exercise, employmente, other daily activities, and adverse pregnancy outcomes. Am J Epidemiol 1996; 143: 211-8.

35. Gottlieb S. Exercise during pregnancy may increase birth weight. BMJ 2001; 322: 193-4.

36. ACOG (American College of Obstetricians and Gynecologists). Committee on Obstetric. Exercise during pregnancy and the postpartum period. Pratice n. ${ }^{\circ} 267$. Am Col Obstet Gynecol 2002; 99: 171-3.

37. Katz VL. Water exercise in pregnancy. Semin Perinatol 1996; 2: 285-91.

38. Camporesi EM. Diving and pregnancy. Semin Perinatol 1996; 20: 292-302.

39. Artal R, Wiswell RA, Drinkwater BL. Orientações de exercícios para a gravidez. In: Artal R, Wiswell RA, Drinkwater BL. O exercício na gravidez. São Paulo: Manole; 1999. p. 299-312.

40. Huch R. Physical activity at altitude in pregnancy. Semin Perinatol 1996; 20: 303-14.

Recebido em 11 de dezembro de 2002

Versão final reapresentada em 5 de março de 2003

Aprovado em 28 de março de 2003 